\title{
Comparação entre medidas implícitas e explícitas na avaliação de casais homoafetivos e heteroafetivos*
}

\section{Comparison between implicit and explicit measures in the evaluation of gay male and heterosexual couples}

\author{
Comparación entre medidas implícitas y explícitas en la evaluación de \\ parejas del mismo sexo y hetero afectivas
}

Maisa Kirchmaier Teixeira' ${ }^{1}$, João Henrique de Almeida², Renato Bortoloti', Edson Massayuki Huziwara'

[1] Universidade Federal de Minas Gerais [2] Universidade Estadual de Londrina I Título abreviado: Avaliação de casais hetero e homoafetivos | Endereço para correspondência: Edson Huziwara. Rua Araripe, 10, apto 1001. Belo Horizonte, MG. CEP 31015-26 | Email: huziwara.edson@gmail.com I doi: $10.18761 /$ PAC.2021. v12.RFT.08

Resumo: Devido às possíveis limitações relacionadas ao uso de medidas explícitas em pesquisas envolvendo questões socialmente sensíveis, o uso de medidas implícitas tem se tornado uma prática recorrente em diversas áreas do conhecimento. Nesse contexto, tornam-se necessários experimentos que comparem os resultados gerados por cada tipo de medida. $\mathrm{O}$ objetivo do presente experimento foi exatamente comparar medidas explícitas e implícitas sobre a avaliação de imagens de casais hétero e homoafetivos em participantes autodeclarados hétero e homossexuais. Para a obtenção da medida explícita, os participantes avaliaram as referidas imagens por meio de uma escala Likert de cinco pontos. Para a obtenção da medida implícita, eles realizaram uma tarefa utilizando o Implicit Relational Assessment Procedure (IRAP). De maneira geral, os resultados do IRAP demonstraram que participantes heterossexuais apresentaram um viés comportamental positivo tanto para fotos de casais heteroafetivos quanto para fotos de casais homoafetivos. Participantes homossexuais, por sua vez, apresentaram um viés comportamental positivo para fotos de casais homoafetivos e foram neutros com relação a fotos de casais heteroafetivos. Por meio da análise dos resultados foi possível observar que as medidas implícitas e explícitas deferiram principalmente para os participantes autodeclarados homossexuais. Tais resultados são discutidos a luz de Teoria das Molduras Relacionais e das possíveis diferenças existentes na história de reforçamento que participantes desses diferentes grupos são expostos.

Palavras-chave: Medidas explícitas; Medidas implícitas; IRAP; Orientação sexual; Jovens adultos.

\footnotetext{
*Esta pesquisa contou com o apoio do Instituto Nacional de Ciência e Tecnologia em Comportamento, Cognição e Ensino (INCT - ECCE), financiado pela Fundação de Amparo à Pesquisa do Estado de São Paulo (FAPESP, processo no 2014/50909-8), pela Coordenação de Aperfeiçoamento de Pessoal de Nível Superior (CAPES, processo nº 88887.136407/2017-00), e pelo Conselho Nacional de Desenvolvimento Científico e Tecnológico (CNPq, processo no 465686/2014-1).
} 


\begin{abstract}
Due to possible limitations related to the use of explicit measures in research involving socially sensitive matters, the use of implicit measures has become a recurrent practice in several areas of knowledge. In this context, experiments which compare results generated by each type of measure become necessary and timely. The major aim of the present experiment was exactly to compare explicit and implicit measures on the evaluation of images of heterosexual and homosexual couples in self-declared heterosexual and gay men participants. For obtaining the explicit measure, the participants evaluated these images using a five-point Likert scale. For obtaining the implicit measure, in turn, they performed a task using the Implicit Relational Assessment Procedure (IRAP). In general, the IRAP results showed that heterosexual participants showed a positive behavioral bias both for photos of hetero-affective couples and for photos of same-sex couples. Gay men participants, in turn, showed a positive behavioral bias for photos of same-sex couples and were neutral with respect to photos of hetero-affective couples. Through the analysis of the results, it was possible to observe that the implicit and explicit measures differed mainly for the self-declared gay men participants. Such results are discussed in the light of the Relational Frame Theory and the possible differences in the reinforcement history of participants in these different groups.
\end{abstract}

Keywords: Explicit measures; Implicit measures; IRAP; Sexual orientation; Young adults.

Resumen: Debido a las posibles limitaciones relacionadas con el uso de medidas explícitas en investigaciones que involucran temas socialmente sensibles, el uso de medidas implícitas se ha convertido en una práctica recurrente en varias áreas del conocimiento. En este contexto, se necesitan experimentos para comparar los resultados generados por cada tipo de medida. El objetivo del presente experimento fue exactamente comparar medidas explícitas e implícitas sobre la evaluación de imágenes de parejas heterosexuales y homosexuales en participantes auto declarados heterosexuales y homosexuales. Para obtener la medida explícita, los participantes evaluaron estas imágenes utilizando una escala Likert de cinco puntos. Para obtener la medida implícita, realizaron una tarea utilizando el Procedimiento de Evaluación Relacional Implícita (IRAP). En general, los resultados del IRAP mostraron que los participantes heterosexuales mostraron un sesgo conductual positivo tanto para las fotos de parejas hetero afectivas como para las fotos de parejas homo afectivas. Los participantes homosexuales, a su vez, mostraron un sesgo conductual positivo para las fotos de parejas del mismo sexo y fueron neutrales con respecto a las fotos de parejas hetero afectivas. A través del análisis de los resultados se pudo observar que las medidas implícitas y explícitas difirieron principalmente para los participantes homosexuales auto declarados. Dichos resultados se discuten a la luz de la Teoría de los marcos relacionales y las posibles diferencias en la historia de reforzamiento a la que están expuestos los participantes de estos diferentes grupos.

Palabras clave: Medidas explícitas; Medidas implícitas; IRAP; Orientación sexual; Adultos jóvenes. 
Temas socialmente sensíveis têm sido frequentemente investigados a partir de métodos que requerem respostas explícitas, como questionários, entrevistas e autoavaliações. Embora tais instrumentos sejam úteis e convenientes especialmente para pesquisas com grandes amostras (Expósito et al., 2015; Nolan et al., 2013), eles podem estar sujeitos a algumas limitações. Afirma-se, por exemplo, que alguns participantes podem omitir sua verdadeira opinião acerca de um tema controverso caso estejam cientes de que essa opinião possa ser considerada como socialmente indesejável (Barnes-Holmes et al., 2006; Bast et al., 2016; Dawson et al., 2009; Nolan et al., 2013; Power et al., 2009; Rabelo et al., 2014). Ou seja, diante de questionários ou outras medidas explícitas, os participantes podem não estar dispostos a expressar acuradamente seus julgamentos. Além disso, também é preciso considerar a possibilidade de eles não terem consciência de vieses implícitos que influenciam seus comportamentos (Cullen \& Barnes-Holmes, 2008). Por esses motivos, a validade dos resultados obtidos a partir de pesquisas que utilizaram os referidos métodos tradicionais é muitas vezes questionada (Bast et al., 2016; Drake et al., 2010; Jellison et al., 2004; Murphy et al., 2014; Power et al., 2009; Rabelo et al., 2014; Rönspies et al., 2015; Snowden et al., 2008).

Como alternativa a esses métodos tradicionais, a investigação de questões socialmente sensíveis tem se utilizado de medidas implícitas de vieses comportamentais, pois se acredita que tais medidas podem reduzir a influência de variáveis intervenientes (Barnes-Holmes et al., 2006, Barnes-Holmes et al., 2010a). O procedimento mais utilizado para a obtenção de medidas implícitas é o Teste de Associação Implícita (IAT), proposto para avaliar associações entre conceitos mais bem estabelecidas na memória dos participantes (Greenwald et al., 1998). No IAT, pede-se aos participantes que categorizem estímulos de interesse com termos positivos e negativos, em blocos sucessivos de tentativas. Especificamente, em um IAT típico, os participantes são solicitados a parear dois estímulos alvo distintos com dois atributos (e.g., alvos: gordo e magro; atributos: atraente e repulsivo). Desempenhos mais rápidos e precisos são comumente relatados quando alvos e atributos mais bem associados para o participante (e.g., magro - atra- ente) requerem uma mesma resposta (e.g., teclar "d") do que quando conceitos não associados (e.g., gordo - atraente) requerem essa mesma resposta (Greenwald et al., 1998). O IAT tornou-se uma alternativa à pesquisa baseada em medidas explícitas de autorrelato que muitas vezes podem gerar vieses nos resultados e interpretações (Maison et al., 2004). Embora seja útil para uma ampla gama de situações, o IAT não fornece informações sobre a natureza ou direção da associação em avaliação (Barnes-Holmes et al., 2010b).

Uma alternativa ao IAT é fornecida pelo Procedimento de Avaliação Relacional Implícita (IRAP; Barnes-Holmes et al., 2010b), originalmente desenvolvido para medir vieses comportamentais relacionados aos mais variados temas. Como exemplo, Nicholson e Barnes-Holmes (2012) utilizaram o IRAP para investigar aversão aranhas. Os participantes foram divididos em dois grupos, com base em escores obtidos por meio da aplicação do Fear of Spiders Questionnaire - FQS (Szymanksi \& O’Donohue, 1995). Um grupo foi formado por indivíduos que relataram baixa aversão a aranhas e o outro grupo por indivíduos que relataram alta aversão. Todos os participantes foram submetidos a um IRAP programado para investigar vieses comportamentais em relação a aranhas. Nesse procedimento, fotografias de aranhas eram apresentadas no topo da tela de um computador, sendo denominadas de "estímulos alvo". Logo abaixo, uma frase era apresentada como "estímulo rótulo". O rótulo apresentado em cada tentativa poderia indicar presença (e.g., "tenho medo") ou ausência (e.g., "posso me aproximar") de medo. Além disso, duas opções de resposta relacionais eram apresentadas nos cantos inferiores da tela (VERDADEIRO e FALSO). Em alguns blocos de tentativas, os participantes eram requisitados a responder de forma a indicar que possuíam aversão a aranhas (i.e., eles eram deveriam escolher "verdadeiro" nas tentativas em que a imagem de uma aranha era apresentada com frases indicativas de medo e escolher "falso" nas tentativas em que a imagem era apresentada com frases indicativas de ausência de medo). Em outros blocos, os participantes eram requisitados a responder de forma a indicar que não possuíam aversão a aranhas (i.e., escolher "falso" diante da combinação aranha e medo e escolher "verdadeiro" diante da combi- 
nação aranha e ausência de medo). O IRAP é baseado na premissa de que, dada uma determinada combinação de estímulos rótulo e alvo, a latência será menor nos blocos em que as respostas forem consistentes com o histórico de aprendizagem do participante quando comparada à latência nos blocos em que as respostas forem inconsistentes.

Finalizado o IRAP, os participantes eram submetidos a uma etapa que envolvia a aproximação controlada de uma tarântula real. Essa aproximação estava dividida em seis passos, dos quais o primeiro passo consistia em permitir que o experimentador abrisse uma porta que dava acesso ao terrário em que a tarântula estava colocada e o sexto passo consistia em colocar a mão no interior do terrário e mantê-la imóvel por $2 \mathrm{~min}$. De acordo com Nicholson e Barnes-Holmes (2012), os resultados obtidos no IRAP permitiram predizer o comportamento exibido na tarefa que envolvia tarântulas reais. Ou seja, os participantes que apresentavam resultados no IRAP indicativos de pouco medo foram os mesmos que atingiram estágios mais avançados de aproximação da tarântula. E ainda, resultados indicativos de muito medo foram apresentados pelos mesmos participantes que não avançaram nos estágios de aproximação da aranha. Com relação aos resultados da medida explícita, sete dentre os 15 participantes que relataram altos níveis de aversão completaram todos os passos de aproximação da tarântula real. Portanto, os resultados obtidos por meio de medidas explícitas parecem ter sido menos precisos quanto comparados aos resultados obtidos no IRAP.

A utilização de medidas implícitas tem sido ferramenta valiosa para o estudo de temas socialmente controversos, tais como o racismo (BarnesHolmes et al., 2010a) e o preconceito étnico (Power et al., 2009), principalmente por obter resultados supostamente mais reveladores do que aqueles obtidos por meio de medidas explícitas. A sexualidade humana se constitui em outro exemplo de tema socialmente sensível no qual medidas implícitas têm sido utilizadas (Anselmi et al., 2013; Cullen \& Barnes-Holmes, 2008; Jellison et al., 2004; Rönspies et al., 2015; Scheel et al., 2011; Timmins et al., 2016). Relacionado a esse tema, estudos com o IRAP podem ser divididos em dois conjuntos consideravelmente distintos entre si. $\mathrm{O}$ primeiro conjunto engloba experimentos com o objetivo de verificar se a orientação sexual dos participantes teria alguma influência nos resultados obtidos por meio do IRAP quando rótulos e alvos especificam a atração sexual exercida por diferentes estímulos (Rönspies et al., 2015; Timmins et al., 2016). No estudo de Timmins et al. (2016), por exemplo, participantes hétero e homossexuais realizavam uma tarefa na qual eram apresentadas fotografias de homens e mulheres como alvos e palavras que remetiam ao apelo sexual exercido por eles (i.e., atrativo, bonito, não atrativo, feio) como rótulos.

Os objetivos do segundo conjunto de experimentos estão mais direcionados para investigar possíveis situações de preconceito social relacionadas a orientação sexual dos indivíduos (Cullen \& Barnes-Holmes, 2008; Scheel et al., 2011). No estudo conduzido por Cullen e Barnes-Holmes (2008), por exemplo, o IRAP apresentou como estímulos rótulo uma de duas palavras escritas em inglês para designar tipos de orientação sexual (i.e., 'Gay' e 'Straight'). Além disso, dois conjuntos de estímulos-alvo foram apresentados; um que compreendia termos negativos (e.g., "anormal" ou "perigoso") e o outro conjunto que compreendia termos positivos (e.g., "normal" ou "seguro"). Participantes hétero e homossexuais foram solicitados a escolher de forma rápida e precisa um de dois termos relacionais (i.e., 'Semelhante' ou 'Oposto'). A análise de dados obtidos no IRAP permitiu observar que os grupos divergiram apenas nas tentativas que apresentavam a palavra 'Gay' como rótulo e os termos negativos como alvos. Enquanto o grupo composto por participantes homossexuais selecionou a opção 'oposto' mais rapidamente que a opção 'semelhante', o grupo composto por participantes heterossexuais produziu um padrão de resultados inverso.

De acordo com Morrison et al. (2005), formas tradicionais de preconceito relacionado a orientação sexual dos indivíduos parecem estar sendo substituídas por um tipo mais sutil de preconceito. Em outras palavras, concepções como 'a homossexualidade é uma corrupção social que precisa ser eliminada' parecem estar menos presentes na sociedade atual. Por outro lado, parecem ser cada vez mais frequentes expressões daquilo que tem sido denominado de homonegatividade moderna (Morrison \& Morrison, 2002), como por 
exemplo: 'homens gays e mulheres lésbicas exigem mudanças sociais desnecessárias, como por exemplo, o direito de se casar' ou ainda 'gays e lésbicas colocam muita ênfase em sua sexualidade e, ao fazê-lo, são culpados por sua própria marginalização'. De acordo com Cullen e Barnes-Holmes (2008), o "viés de negatividade" observado com o IRAP e o surgimento dessas formas mais sutis de preconceito sugerem que a vigilância contínua e pesquisas sobre esse tema continuam sendo particularmente importantes.

Relacionada a essas formas sutis de preconceito, o presente estudo teve como objetivo verificar se fotografias de casais homoafetivos e heteroafetivos em situações românticas seriam avaliadas de forma diferente por pessoas autodeclaradas hétero e homossexuais. Foram obtidas medidas implícitas, utilizando o IRAP, e medidas explícitas, por meio de uma escala Likert. Ao comparar os resultados encontrados nas diferentes medidas, foi possível verificar se o padrão de respostas explícitas coincidiu ou não com o padrão de respostas encontrados nos testes de viés comportamental. Os resultados advindos da realização desse experimento ampliam a gama de temas socialmente relevantes para os quais o IRAP tem sido utilizado, pois, estudos acerca de viés comportamental relacionadas à orientação sexual dos indivíduos utilizando esse procedimento ainda são escassos (Cullen \& Barnes-Holmes, 2008; Rönspies et al., 2015). O estudo e desenvolvimento de medidas apropriadas para investigação de tais temas pode também ser de grande importância prática, uma vez que o viés comportamental dos indivíduos pode influenciar ações discriminatórias contra populações socialmente desfavorecidas (Anselmi et al., 2013; Cullen \& Barnes-Holmes, 2008; Jellison et al., 2004; Rönspies et al., 2015).

\section{Método}

\section{Participantes}

Inicialmente, foram coletados dados de 58 participantes. Os dados de 23 deles, no entanto, não foram incluídos na análise final em virtude de uma das seguintes razões: ou eles não atingiram os critérios de tempo e acurácia exigidos durante os blocos de treino do IRAP ou eles não mantiveram esses crité- rios em, pelo menos, quatro dos seis blocos de teste do IRAP. Portanto, 35 homens com idades entre 18 e 36 anos $(M=24,7 ; D P=5,3)$ compuseram a amostra analisada no presente estudo. Tais participantes, de acordo com uma autodeclaração, foram divididos em dois grupos, sendo 18 autodeclarados homossexuais e 17 autodeclarados heterossexuais. A divulgação da pesquisa e captação de participantes ocorreu por meio de anúncios publicados em um campus universitário e por convites pessoais. Os participantes leram um termo de consentimento com informações sobre a pesquisa antes do início do experimento. Ao final de sua participação, eles foram informados sobre os objetivos do experimento e as questões processuais em consideração. Eles não receberam pagamento ou compensação por participar da pesquisa.

\section{Situação e Equipamentos}

As atividades foram realizadas em uma sala de $28 \mathrm{~m}^{2}$ localizada em uma instituição de ensino superior, especialmente preparada para tal finalidade, em condições adequadas para evitar interrupções desnecessárias ou ruídos estranhos à realização do experimento.

Para aplicação do procedimento computadorizado IRAP foi utilizado o programa GO-IRAP instalado em um computador desktop com monitor de 15". O software foi empregado para apresentar os estímulos, liberar as consequências programadas e registrar as respostas emitidas pelos participantes.

\section{Procedimento}

\section{Medida Explícita}

O material apresentado para obtenção da medida explícita continha cinco páginas, sendo a primeira página destinada a apresentar as instruções para a realização da tarefa e as quatro páginas seguintes destinadas a apresentar as escalas a serem preenchidas. No topo de cada página foram apresentadas separadamente quatro fotografias ${ }^{1}$ em tamanho $10 \times 14 \mathrm{~cm}$, sendo duas de casais homoafetivos e duas de casais heteroafetivos. Abaixo

1 As fotografias utilizadas no presente experimento foram adquiridas nos seguintes sites de comercialização de imagens: www.dreamstime.com, www.pexels.com e www.twenty20.com. 
de cada fotografia era apresentado um conjunto de quatro escalas bipolares. Cada uma das escalas apresentava pares de adjetivos opostos (i.e., bom-ruim, agradável-desagradável, normal-estranho e bonito-feio) sendo apresentados nas extremidades direta e esquerda da folha. Além disso, um conjunto de cinco pontos dispostos lado-a-lado, na horizontal, era apresentado entre os adjetivos. O participante era solicitado a marcar um dos pontos. Para fins de análise, cada ponto assinalado correspondia a um valor, que variava de -2 a +2 . Atribuições positivas relacionadas à fotografia deveriam ser expressas pela seleção de pontos próximos aos adjetivos positivos, da mesma forma que atribuições negativas deveriam ser expressas pela seleção de pontos próximos aos adjetivos negativos. A escolha do ponto central, equidistante de ambos os adjetivos e correspondente ao ponto 0 , era interpretado como se o conceito estivesse igualmente relacionado a ambos os adjetivos ou, então, como se o conceito não estivesse relacionado a nenhum dos adjetivos do par sob consideração.

\section{Medida Implícita de Viés Comportamental}

Após preencher a escala Likert, os participantes realizavam o procedimento para a obtenção da medida do viés comportamental. Antes de iniciar a tarefa, as seguintes instruções eram apresentadas na tela do computador: "Olá! Nesta atividade, você irá visualizar uma imagem, uma palavra e, como opções de resposta, "verdadeiro" ou "falso". Você só precisa fazer associação entre a imagem e a palavra, de acordo com a regra que lhe for dada anteriormente, dizendo se a associação é verdadeira ou falsa. A regra sobre essa associação será dada pelo programa e mudará ao longo do tempo. Para responder, você deverá pressionar a tecla " $D$ " para verdadeiro e "K" para falso. Se você acertar, aparecerá uma nova tentativa. Se você errar, um "X" vermelho irá aparecer, portanto responda novamente. O MAIS IMPORTANTE É RESPONDER O MAIS RÁPIDO POSSÍVEL E TENTAR NÃO COMETER ERROS! Coloque os dedos sobre as teclas "D" e "K" e aperte espaço para continuar".

Cada tentativa apresentava um estímulo rótulo, um estímulo alvo e duas opções de resposta. Os estímulos rótulo eram as mesmas fotografias de ca- sais homoafetivos e heteroafetivos usadas na escala Likert. Os estímulos alvo, que apareciam simultaneamente às fotografias, eram palavras com conteúdo semântico positivo, como "agradável", "bom", "bonito", "normal" ou palavras com conteúdo semântico negativo, como "desagradável", "ruim", "feio" e "estranho". Os participantes eram requisitados a escolher uma de duas opções de resposta, que apareceram nos cantos inferiores esquerdo e direito da tela. A posição das opções de resposta permaneceu inalterado durante todo o procedimento.

\section{Fase de Treino}

A fase de treino apresentava blocos com 24 tentativas. Nos blocos denominados pró-hetero, a seguinte instrução era apresentada aos participantes: "Responda associando HETEROSSEXUAL a PALAVRAS POSITIVAS e HOMOSSEXUAL a PALAVRAS NEGATIVAS”. Portanto, os participantes eram requeridos a escolher a opção "verdadeiro" nas tentativas que apresentavam casais heteroafetivos como rótulo e palavras de conteúdo semântico positivo como alvo, assim como nas tentativas que apresentavam casais homoafetivos como rótulo e palavras de conteúdo semântico negativo como alvo. Além disso, nesses mesmos blocos, eles deveriam escolher a opção "falso" nas tentativas que apresentavam casais heteroafetivos como rótulo e palavras de conteúdo semântico negativo como alvo e, também, nas tentativas que apresentavam fotografias de casais homoafetivos como rótulo e palavras de conteúdo semântico negativo como alvo.

Nos blocos denominados pró-homo, por sua vez, os participantes eram solicitados a responder de forma exatamente contrária. As seguintes instruções eram apresentadas: "Responda associando HOMOSSEXUAL a PALAVRAS POSITIVAS e HETEROSSEXUAL a PALAVRAS NEGATIVAS". Assim, eles deveriam escolher a opção "verdadeiro" diante das combinações casais homoafetivos-palavras positivas e casais heteroafetivos-palavras negativas. E ainda, escolher a opção "falso" diante das combinações casais homoafetivos-palavras negativas e casais heteroafetivos-palavras positivas. A Figura 1 apresenta exemplos das quatro combinações rótulo e alvo possíveis, assim como o padrão de respostas exigido em cada um dos blocos. 

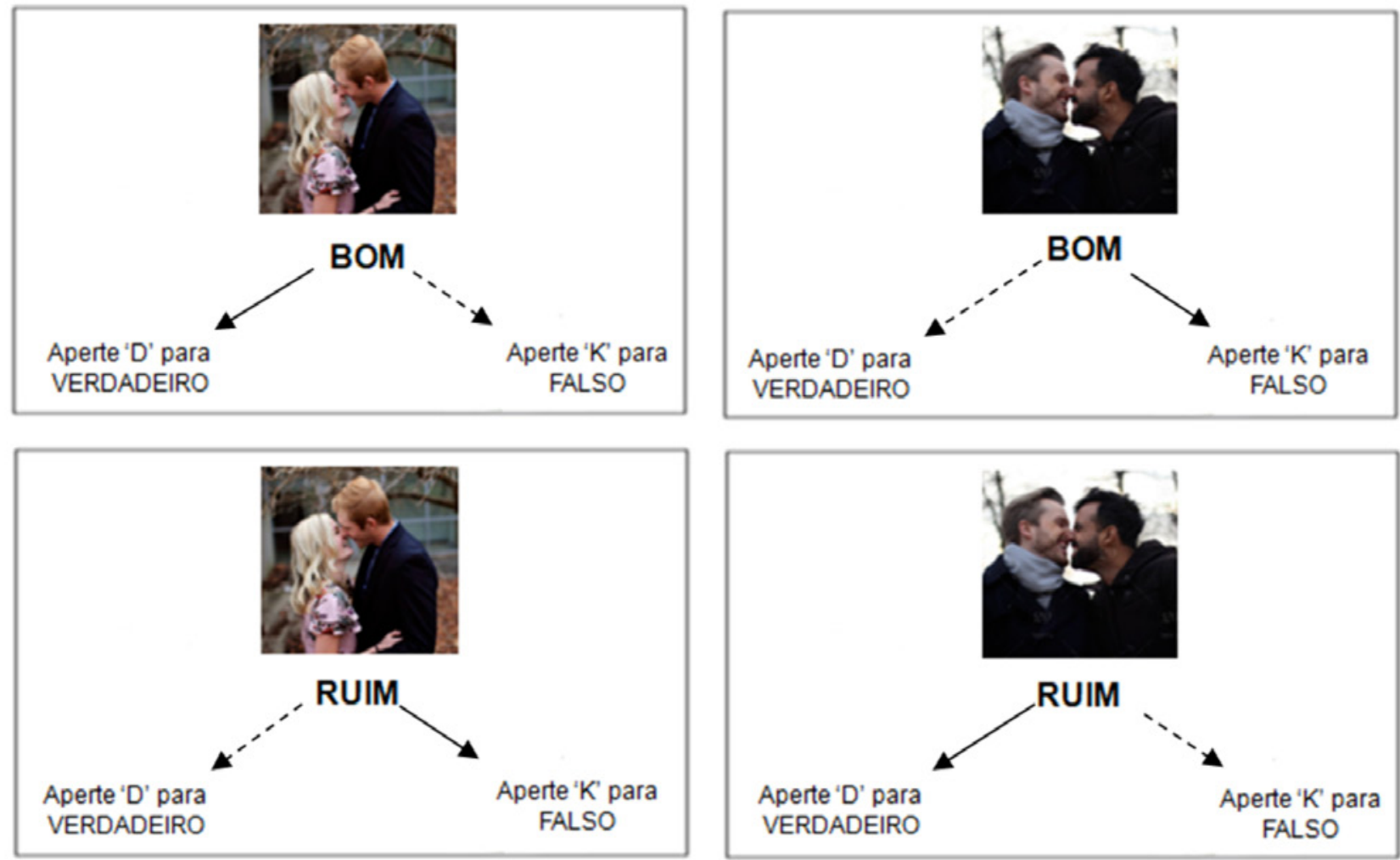

Figura 1. Exemplos de tentativas e padrões de respostas exigidos. As setas com linhas contínuas apresentam os padrões de respostas exigidos nos blocos pró-hetero e as setas com linhas tracejadas apresentam os padrões de respostas exigidos nos blocos pró-homo.

Em ambos os tipos de blocos, respostas corretas eram consequenciadas com a apresentação de uma nova tentativa após um intervalo de $400 \mathrm{~ms}$. Respostas incorretas eram consequenciadas pela apresentação de X vermelho no centro da tela. Para seguir para a próxima tentativa após emitir uma resposta incorreta, o participante deveria selecionar a opção correta para a tentativa apresentada.

Nessa etapa de treinos, o participante era submetido a no mínimo dois e no máximo seis blocos, sempre alternados em pares pró-hetero e pró-homo. Para prosseguir a fase de testes, o participante precisaria atingir nos dois tipos de blocos os critérios de acurácia igual ou superior a $80 \%$ de respostas corretas e média de latência igual ou inferior a $2.000 \mathrm{~ms}$. A quantidade de blocos dependia do desempenho do participante e da quantidade de blocos que precisaria para aprender a tarefa e atingir aos critérios exigidos. Caso o participante não atingisse os critérios após realizar seis blocos de treino, sua participação no experimento era encerrada.

\section{Fase de Teste}

Após a etapa de treino, os participantes foram submetidos a seis blocos de 24 tentativas, também alternados em pares pró-hetero e pró-homo. Nessa etapa, exatamente como acontecia na fase de treinos, os participantes também recebiam as instruções com as regras vigentes em cada bloco e a posição das opções de respostas também se manteve inalterada. Importante salientar que apenas os dados obtidos na fase de teste foram utilizados para efeitos de análise e comparação de latências.

\section{Resultados}

\section{Medida Explícita: Escala Likert}

Como pode ser observado na Figura 2, a mediana do escore geral da escala Likert para participantes heterossexuais na avaliação das imagens de casais heteroafetivos foi de $M D=1,00$. A mediana da avaliação deste mesmo grupo em relação às imagens 


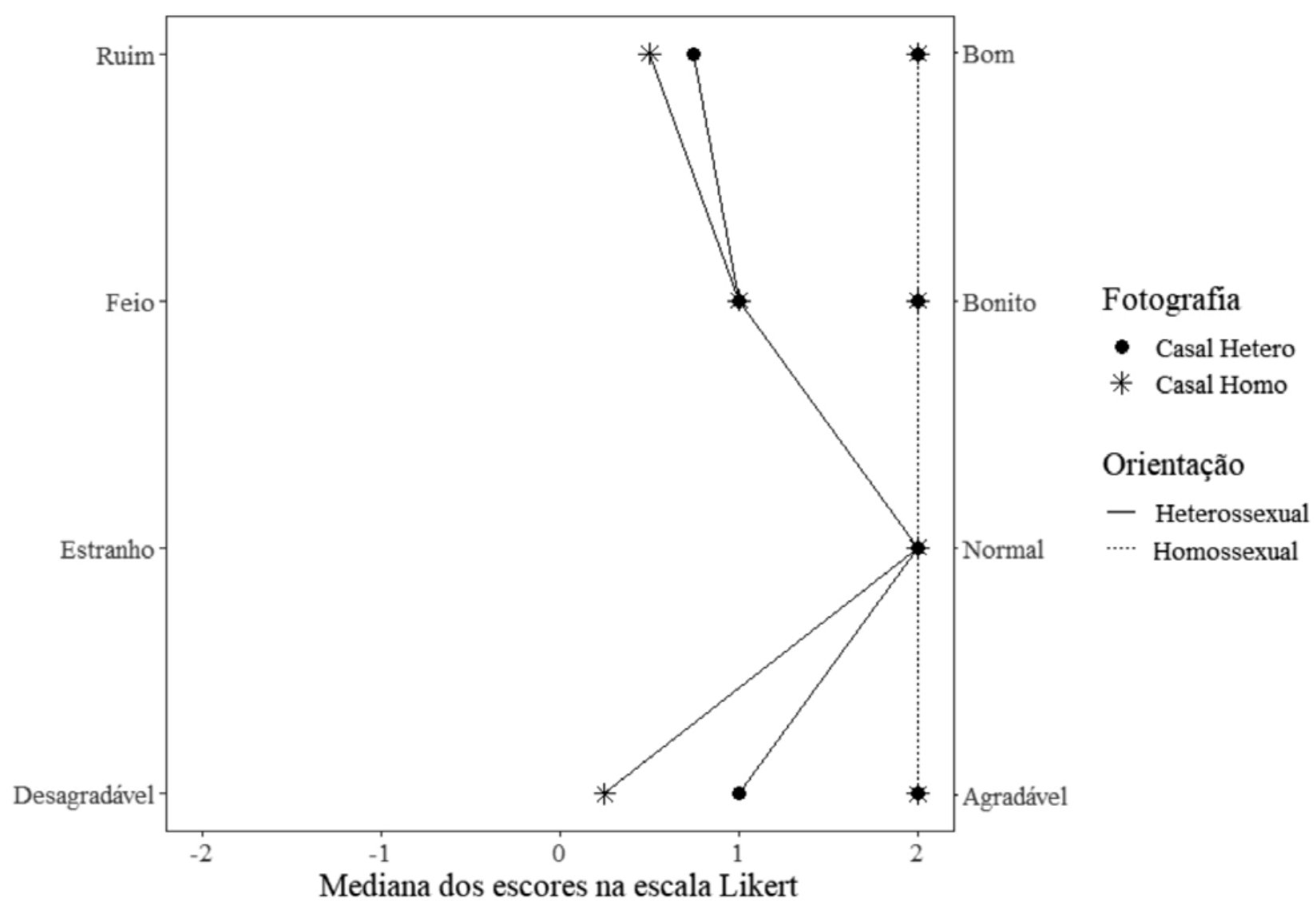

Figura 2. Medianas das escalas Likert de participantes heterossexuais (linhas contínuas) e homossexuais (linhas tracejadas) na avaliação de fotografias de heterossexuais e homossexuais.

de casais homoafetivos foi $M D=0,88$. Para o grupo de homossexuais, a mediana da avaliação das imagens de casais heteroafetivos foi de $M D=1,88$ e para imagens de casais homoafetivos foi de $M D$ $=2,00$. Os dados analisados mostram que ambos os grupos tenderam a avaliar positivamente tanto as fotos de casais heteroafetivos quanto homoafetivos, sendo que os participantes homossexuais apresentaram valores maiores e com menores desvios padrão (i.e., menor dispersão) quando comparados aos participantes heterossexuais.

Uma vez que as medidas explícitas apresentaram uma distribuição assimétrica, violando o pressuposto de normalidade, foi utilizado o teste não paramétrico Mann-Whitney para comparação dos grupos. As atribuições realizadas nas escalas Likert considerando todos os adjetivos apresentados agradável/desagradável, feio/bonito, ruim/bom e estranho/normal) foi comparada entre participantes considerando tanto o tipo de foto (casais hetero e casais homo) quanto à orientação sexual (participante hetero e homossexual). Verificaram-se diferenças estatisticamente significativas entre participantes hetero e homossexuais, tanto no escore das avaliações das fotografias de casais heteroafetivos $(U=1529, p<0.0001)$ quanto de homoafetivos ( $U$ $=1020, p<0,0001)$. Mais especificamente, a mediana da avaliação de heterossexuais foi significativamente menor do que a avaliação de homossexuais para os dois tipos de fotografias. 


\section{Medida Implícita: IRAP}

Em termos gerais, os resultados obtidos no IRAP são analisados a partir da diferença nas latências de resposta obtidas nos blocos pró-hetero e pró-homo para cada possível combinação entre rótulo e alvo, sendo a latência definida como o tempo decorrido entre a apresentação dos estímulos e a emissão da resposta de escolha. Mais especificamente, o valor da latência para selecionar a opção "verdadeiro" diante da combinação hétero-positivo (i.e., bloco pró-hetero) foi subtraída do valor da latência para selecionar a opção "falso" diante dessa mesma combinação (i.e., bloco pró-homo). Esse mesmo cálculo foi realizado para todas as demais combinações rótulo-alvo. Os valores absolutos das latências foram processados usando o algoritmo D-IRAP, disponível no software GO-IRAP (para descrição dos passos para obtenção do D-IRAP, ver Dawson, Barnes-Holmes, Gore, \& Gresswell, 2009) para controlar diferenças individuais nos tempos de resposta associadas a fatores desconhecidos.
Nesse contexto, os valores positivos do D-IRAP seriam obtidos caso os participantes respondessem mais rapidamente às tentativas dos blocos pró-hetero do que dos blocos pró-homo. Valores negativos, por sua vez, seriam obtidos caso eles respondessem mais rapidamente às tentativas dos blocos pró-homo do que dos blocos pró-hetero. Escores D-IRAP referentes a cada combinação rótulo-alvo foram calculados para os participantes heterossexuais e homossexuais separadamente, possibilitando a comparação entre os resultados dos dois grupos.

A Figura 3 apresenta a média dos escores D-IRAP encontrados para os participantes de ambos os grupos nas diferentes combinações modelo-alvo. As barras pretas representam resultados D-IRAP de participantes heterossexuais e as barras cinzas representam os resultados D-IRAP dos participantes homossexuais. Um teste $t$ foi realizado com o objetivo de avaliar se as médias dos escores D-IRAP encontrados em cada tipo de tentativa eram estatisticamente diferentes de zero. O asteris-

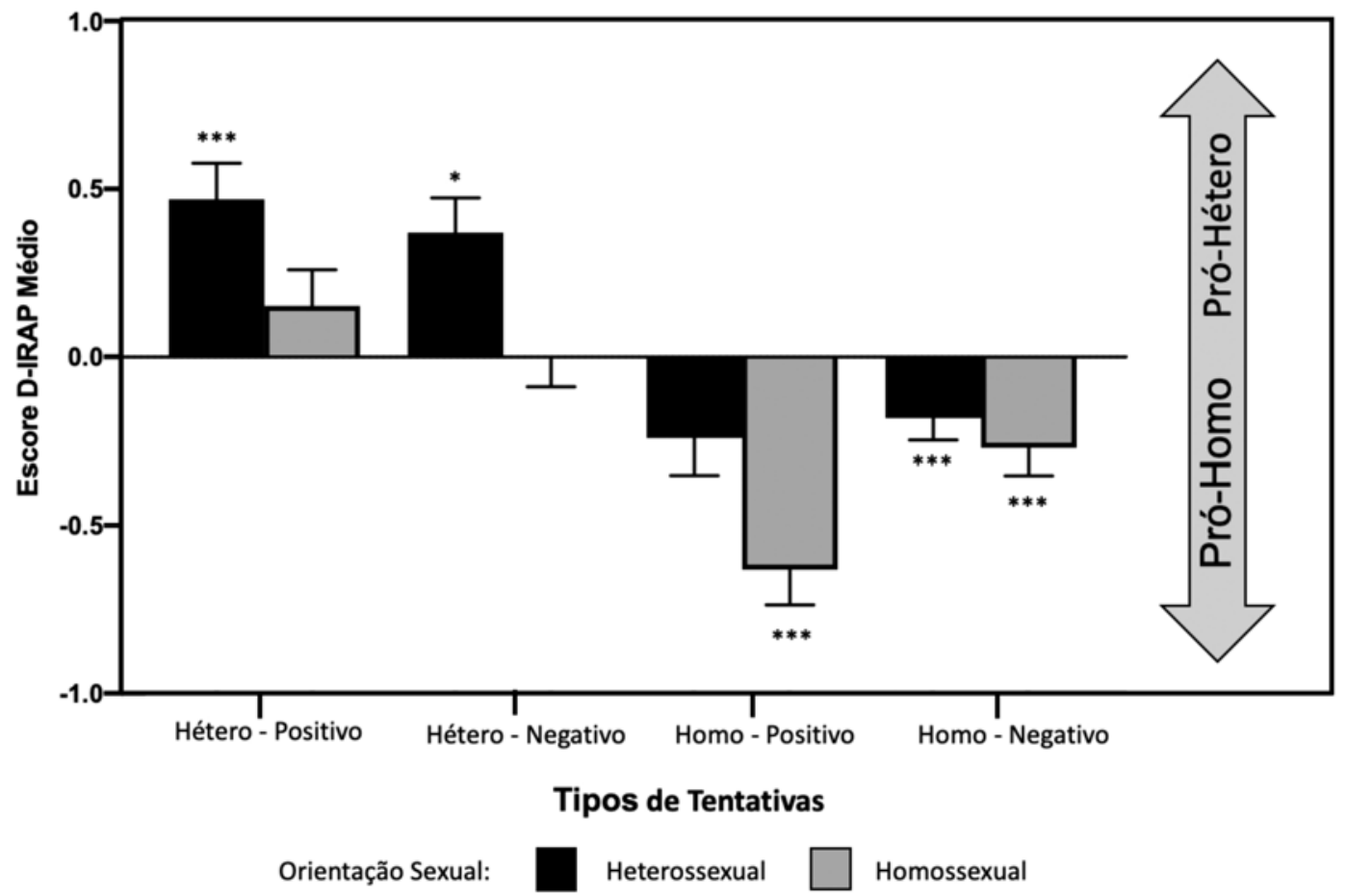

Figura 3. Escores D-IRAP para os diferentes tipos de tentativas separadas por orientação sexual. Cada um dos valores apresenta barras de erro que correspondem ao Erro Médio Padrão. 
co indica a magnitude do valor-p encontrado no teste t. Um asterisco $\left(^{*}\right)$ indica o valor $p<0,05$, dois asteriscos $\left(^{\star *}\right)$ indicam o valor $p<0,01$ e três asteris$\cos \left({ }^{* * *}\right)$ indicam o valor $p<0,001$.

Em relação ao grupo composto por homens heterossexuais, pode-se considerar que as médias dos escores D-IRAP encontradas nas tentativas HeteroPositivo $[t(17)=4,107, p<0,001]$ Hetero-Negativo $[t(17)=2,699, p=0,015]$, e Homo-Negativo $[t(17)=-$ $2,9028, p=0,009]$ foram estatisticamente diferentes de zero. As médias encontradas nas tentativas Homo-Positivo $[t(17)=-1,7479 ; p=0,098]$ não foram estatisticamente diferentes de zero.

Para o grupo composto por homens homossexuais pode-se considerar que a média dos escores D-IRAP para as tentativas Homo-Positivo $[t(16)=-$ $6,433 ; p<0,001]$ e Homo-Negativo $[t(16)=-4,1805$; $p<0,001$ [ foram significativamente diferentes de zero. Os valores encontrados para as tentativas Hetero-positivo $[t(16)=0,7696, p=0,452]$ e Heteronegativo $[t(16)=-0,45191, p=0,657]$ não foram estatisticamente diferentes de zero.

\section{Correlação medida implícita de viés} comportamental e medida explícita

Para realizar uma análise de correlação entre as medidas de viés comportamental e explícitas, considerando que as medidas explícitas possuem uma distribuição assimétrica e as medidas implícitas possuem uma distribuição normal, foi utilizado o teste estatístico não paramétrico Coeficiente de correlação tau-b de Kendall. Os escores obtidos nas medidas implícitas e explícitas foram separados considerando a orientação sexual dos participantes, as imagens apresentadas e o conteúdo semântico das palavras exibidas na medida implícita.

Tanto para o grupo composto por heterossexuais quanto por homossexuais, os escores D-IRAP das tentativas com imagens de casais heteroafetivos foram correlacionados com os escores da escala Likert que também apresentavam imagens de casais heteroafetivos. Uma correlação moderada e significativa entre essas duas medidas foi encontrada apenas para os participantes heterossexuais, $|\tau b|=0,38$; $p<0,05$. Mais especificamente, quanto maior a pontuação na escala Likert, maior também foi o resultado do D-IRAP indicando um viés comportamental pró-heterossexuais.
De forma semelhante, para ambos os grupos, os escores D-IRAP foram correlacionados com os escores da escala Likert para a situações nas quais casais homoafetivos eram apresentados. A exemplo do ocorrido anteriormente, foi possível uma correlação moderada e significativa entre os escores apenas para os participantes heterossexuais $|\tau b|=-0,48$; $p<0,05$. O valor da correlação nesse caso apresenta valor negativo devido ao modo como os dados do IRAP foram apresentados. Mais especificamente, escores negativos apresentados na Figura 3 representam viés comportamental pró-homossexuais. Assim sendo, para que haja uma concordância entre as medidas, é preciso que quanto mais positivas forem as avaliações feitas na escala Likert, mais negativos deverão ser os escores do D-IRAP, resultando em um valor correlacional negativo (i.e., com sinais invertidos). Em resumo, a correlação indica que quanto maior a pontuação na escala Likert para imagens de casais homoafetivos, maior também foi o resultado do D-IRAP indicando um viés comportamental pró-homossexuais.

\section{Discussão}

O presente trabalho teve como objetivo investigar, a partir da obtenção de medidas explícitas e implícitas, atitudes relacionadas a imagens de casais hétero e homoafetivos em participantes autodeclarados hétero e homossexuais. Para a obtenção da medida explícita, os participantes avaliaram imagens de casais hétero e homoafetivos em contextos românticos por meio de uma escala Likert. Para a obtenção da medida implícita de viés comportamental, eles realizaram uma tarefa IRAP com essas mesmas imagens e palavras de conteúdo semântico positivo e negativo. A análise dos resultados obtidos por meio da medida explícita indicou que tanto participantes hétero quanto homossexuais avaliaram positivamente casais homo e héteroafetivos, porém, o valor médio atribuído a ambos os tipos de casais por participantes homossexuais foi superior quando comparado ao valor atribuído por participantes heterossexuais e essa diferença foi estatisticamente significativa. Com a medida implícita, foi possível observar que participantes heterossexuais apresentaram viés comportamental favorável tan- 
to a imagens de casais héteroafetivos quanto de casais homoafetivos. Participantes homossexuais, por sua vez, apresentaram viés comportamental favorável a imagens casais homoafetivos, mas foram neutros ao avaliar imagens de casais héteroafetivos. Finalmente, de acordo com o Coeficiente de correlação tau-b de Kendall, foram encontradas correlações entre os escores D-IRAP e os escores da Escala Likert apenas para as medidas apresentadas pelo grupo de participantes heterossexuais.

A partir dos conceitos apresentados no modelo REC (sigla para Relational Elaboration and Coherence), o qual foi proposto e desenvolvido no âmbito da Teoria das Molduras Relacionais (Barnes-Holmes et al., 2010b; Hughes et al., 2012), é possível entender diferenças entre os resultados das medidas explícitas e implícitas. De acordo com as proposições desse modelo, respostas relacionais podem ser classificadas de acordo com a janela temporal na qual ocorrem. Assim, respostas emitidas em intervalos curtos são chamadas de BIRR (sigla para Brief and Immediate Relacional Response) e respostas emitidas em intervalos relativamente longos são chamadas de EERR (sigla para Extended and Elaborated Relacional Response). Curiosamente, o tipo de procedimento utilizado para a obtenção da medida irá determinar o tipo de resposta relacional que será acessada, nomeadamente, procedimento com exigência de restrição temporal, como o IRAP, são indicados para acessar BIRRs, enquanto procedimentos sem restrição temporal para emissão da resposta, como questionários e escalas, são indicados para acessar EERRs (Hughes et al., 2012; Expósito et al., 2015).

No contexto do presente experimento, as diferenças nas medidas implícitas e explícitas apresentadas por participantes do grupo homossexual poderiam ser explicadas pelo fato de as respostas emitidas durante a avaliação explícita terem ficado sob controle daquilo que eles consideravam ser mais socialmente aceito (i.e., respostas com altos níveis de complexidade e derivação), ao invés de serem controlados por sua história direta e pessoal de reforçamento (i.e., respostas com baixos níveis de complexidade e derivação). Essa condição de controle deve ter sido invertida durante a realização do IRAP, momento no qual eles foram controlados pela história direta de reforçamento. Além disso, tais diferenças não foram apresentadas por participantes do grupo heterossexual supostamente pelo fato de não haver conflito entre as relações socialmente aceitas e a história pessoal de reforçamento. Por conta disso, parece ter havido uma convergência nas medidas implícitas e explícitas para participantes desse segundo grupo.

Em virtude da similaridade dos procedimentos experimentais, os resultados do presente experimento podem ser comparados com os descritos por Cullen e Barnes-Holmes (2008). Por exemplo, nas tentativas em que a palavra 'Gay' era apresentada juntamente com termos negativos, participantes homossexuais apresentaram um viés pró-homo (i.e., selecionaram a opção 'oposto' mais rapidamente que a opção 'semelhante') enquanto participantes heterossexuais apresentaram um viés anti-homo (i.e., selecionaram a opção 'semelhante' mais rapidamente que a opção 'oposto'). No presente experimento, nas tentativas que apresentavam fotografias de casais homoafetivas juntamente com termos negativos tanto participantes hétero quanto homossexuais apresentaram um viés pró-homo (i.e., selecionaram a opção 'falso' mais rapidamente que a opção 'verdadeiro'). Em que pese possíveis efeitos de se utilizar fotografias ao invés de palavras e opções de respostas relacionais distintas, os resultados do presente experimento parecem diferir do estudo anterior ao indicar que participantes heterossexuais possuem viés favorável no que se refere a avaliação de imagens casais hetero e homoafetivos. Embora possamos nos alegrar com tais descobertas, algumas considerações devem ser pontuadas. Primeiramente, os participantes selecionados para este estudo foram estudantes de graduação e pós-graduação de uma faculdade de uma grande cidade do sudeste, o que possibilita que estes participantes tenham contato, de forma direta ou indireta, com políticas públicas favoráveis a aceitação e heterogeneidade das diferentes orientações sexuais. Assim, esses resultados podem não representar de forma acurada aquilo que seria encontrado se esse mesmo procedimento fosse aplicado com outras populações ou mesmo com universitários de diferentes localidades. Uma pesquisa realizada pelo Ministério dos Direitos Humanos buscou investigar dados sobre a violência contra a população LGBT brasileira no ano de 2016 (Silva, 2017). Os 
resultados revelaram que a maior parte das agressões contra essa população ocorre na casa da vítima, $27,4 \%$, ou na rua, $23,2 \%$, enquanto as agressões no ambiente escolar e universitário representam apenas $2,4 \%$. Em relação à faixa etária dos agressores, a maior parte possui entre 25 a 30 anos, isto é $13,3 \%$, seguidas de $9,1 \%$ que estão entre 36 e 40 anos. Esses dados nos permitem supor que, caso a atual pesquisa fosse realizada fora do contexto universitário, provavelmente os resultados seriam diferentes. Além disso, a idade média dos participantes dessa pesquisa foi de 25,62 anos para participantes heterossexuais e de 23,38 anos para participantes homossexuais. Também com relação a essa variável, se este estudo fosse realizado com pessoas de idades mais avançadas, os resultados encontrados também poderiam ser outros.

Como dito anteriormente, estudos acerca de viés comportamental relacionadas à orientação sexual dos indivíduos utilizando o IRAP ainda são limitados (Cullen \& Barnes-Holmes; 2008; Rönspies et al., 2015), porém uma quantidade considerável de experimentos relacionados a essa temática utilizando outros procedimentos para medir atitudes implícitas foram realizados anteriormente (Anselmi et al., 3013; Breen \& Karpinski, 2013; Jellison et al., 2004; Snowden et al., 2008). Nessas pesquisas anteriores, escalas e inventários para avaliação da orientação sexual dos participantes foram utilizadas para realizar a divisão de grupos (Steffens \& Buchner, 2003; Steffens, 2005). A Escala Kinsey (do inglês Kinsey Scale) utilizada nos experimentos de Steffens e Buchner (2003) e Steffens (2005), por exemplo, investiga aspectos relacionados à vida sexual e preferências do participante. O questionário apresenta perguntas sobre fantasias sexuais, preferências em relação a parceiros, se o participante pode se imaginar tendo experiências sexuais com pessoas do sexo oposto ou do mesmo sexo e se o participante é ou não aberto a viver novas experiências sexuais. Em geral, apenas os participantes para os quais a classificação indica exclusivamente heterossexual ou exclusivamente homossexual são selecionados para fazer parte do experimento.

$\mathrm{Na}$ presente pesquisa, utilizamos apenas o autorrelato para separar os participantes em grupos, um critério supostamente menos rígidos do que aqueles utilizados anteriormente. Portanto, os re- sultados dos participantes do grupo heterossexual, o qual não apresentou diferenças entre medidas explícitas e implícitas, podem, ao menos em parte, ser explicados por esse critério menos rígido ora adotado. Por outro lado, a adoção de tal critério também pode ser considerada um avanço no sentido de que essa população não classificada nos extremos nos inventários de avaliação de orientação sexual, em geral, era excluída da amostra experimental.

Como mencionado anteriormente, as formas tradicionais parecem dar espaço para formas mais sutis, porém igualmente prejudicais de preconceito baseado na orientação sexual dos indivíduos (Cullen \& Barnes-Holmes, 2008; Morrison et al., 2005; Morrison \& Morrison, 2002). Dito de outra forma, pode não ser socialmente aceitável expressar opiniões que indiquem negatividade em relação a gays e lésbicas, mas o viés de negatividade pode fazer com que sejamos mais sensíveis às características negativas presentes em um indivíduo gay do que em um indivíduo heterossexual (Cullen \& Barnes-Holmes, 2008). Assim sendo, justificam-se os esforços no sentido de continuarmos a produzir conhecimentos sobre as mais diferentes formas de preconceito ainda presentes em nossa sociedade. A criação de estratégias eficazes no combate ao preconceito envolve necessariamente o estudo aprofundado do fenômeno em questão. Além disso, também é preciso considerar que a sociedade contemporânea contempla novas e diversas formas de orientação sexual para além das estudadas até esse momento. Portanto, estudo futuros poderiam contemplar diferentes orientações sexuais como, por exemplo, a bissexualidade ou a assexualidade entre outras.

Em resumo, acreditamos que os resultados encontrados no presente experimento contribuem de forma significativa para o avanço no estudo do viés comportamental dos indivíduos em temas socialmente sensíveis. As divergências encontradas entre medidas implícitas e explícitas descritas para os participantes do grupo homossexual ilustram a importância do uso das duas medidas. Os resultados também se fazem importantes uma vez que aumentam gama de temas estudados com IRAP e demonstram a eficácia do instrumento para o estudo de temas relacionados à orientação sexual. 


\section{Referências}

Anselmi, P., Vianello, M., Voci, A., \& Robusto, E. (2013). Implicit sexual attitude of heterosexual, gay, and bisexual individuals: Disentangling the contribution of specific associations to the overall measure. Plos One, 8, 1-6. doi: 10.1371/journal.pone.0078990

Barnes-Holmes, D., Barnes-Holmes, Y., Power, P., Hayden, E., Milne, R., \& Stewart, I. (2006). Do you really know what our believe? Developing the Implicit Relational Assessment Procedure (IRAP) as a direct measure of implicit beliefs. The Irish Psychologist, 32, 1-38.

Barnes-Holmes, D., Barnes-Holmes,Y., Stewart, I., \& Boles, S. (2010a). A sketch of the Implicit Relational Assessment Procedure (IRAP) and the Relational Elaboration and Coherence (REC) model. The Psychological Record, 60, 527542. doi: https://doi.org/10.1007/BF03395726

Barnes-Holmes, D., Murphy, A., Barnes-Holmes, Y., \& Stewart, I. (2010b). The Implicit Relational Assessment Procedure (IRAP): Exploring the impact of private versus public contexts and the response latency criterion on pro-white and anti-black stereotyping among white Irish individuals. The Psychological Record, 60, 57-66. doi: https://doi.org/10.1007/BF03395694

Barnes-Holmes, D., Waldron, D., Barnes-Holmes, Y., \& Stewart, I. (2009). Testing the validity of the Implicit Relational Assessment Procedure (IRAP) and the Implicit Association Test (IAT): Measuring attitudes towards Dublin and country life in Ireland. The Psychological Record, 59, 389-406. doi: https://doi.org/10.1007/ BF03395671

Bast, D. F., Linhares, I. M. P., Gomes, C., Kovac, R., \& Barnes-Holmes, D. (2016). The Implicit Relational Assessment Procedure (IRAP) as a measure of self-forgiveness: The impact of a training history in clinical behavior analysis. The Psychological Record, 66, 177-190. doi: https://doi.org/10.1007/s40732-016-0162-7

Breen, A. B., \& Karpinski, A. (2013). Implicit and explicit attitudes toward gay males and lesbians among heterosexual males and females. The Journal of Social Psychology, 153, 351-374. doi: 10.1080/00224545.2012.739581
Cullen, C. \& Barnes-Holmes, D. (2008). Implicit pride and prejudice: A heterosexual phenomenon? Em: T. G. Morrison \& M. A. Morrison (Eds.). The Psychology of Modern Prejudice, 195-223. New York: Nova Science Publishers.

Dawson, D. L., Barnes-Holmes, D., Gresswell, D. M., Hart, A. J., \& Gore, N. J. (2009). Assessing the implicit beliefs of sexual offenders using the Implicit Relational Assessment Procedure: A first study. Sexual Abuse: A Journal of Research and Treatment, 21, 57-75. doi: $10.1177 / 1079063208326928$

Drake, C. E., Kellum K. K., Wilson K. G., Luoma J. B., Weinstein J. H., \& Adams C. H. (2010). Examining the Implicit Relational Assessment Procedure: Four preliminary studies. The Psychological Record, 60, 81-86. doi: https://doi. org/10.1007/BF03395695

Expósito, P. M., López, M. H., \& Valverde, M. R. (2015). Assessment of implicit anti-fat and pro-slim attitudes in young women using the Implicit Relational Assessment Procedure. Internacional Journal of Psychology and Psychological Therapy, 15, 17-32.

Greenwald, A., McGhee, E., \& Schwartz, J. (1998). Measuring individual differences in implicit cognition: The Implicit Association Test. Journal of Personality and Social Psychology, 74, 1464-1480. doi: 10.1037//0022-3514.74.6.1464

Hughes, S., Barnes-Holmes, D., \& Vahey, N. (2012). Holding on to our functional roots when exploring new intellectual islands: A voyage through implicit cognition research. Journal of Contextual Behavioral Science, 1, 17-38. doi: https://doi.org/10.1016/j.jcbs.2012.09.003

Jellison, W. A., McConnell, A. R., \& Gabriel, S. (2004). Implicit and explicit measures of sexual orientation attitudes: In group preferences and related behaviors and beliefs among gay and straight men. Personality and Social Psychology Bulletin, 30, 629-642. https://doi. org/10.1177/0146167203262076

Maison, D., Greenwald, A., \& Bruin, R. (2004). Predictive validity of the Implicit Association Test in studies of brands, consumer attitudes, and behavior. Journal of Consumer Psychology, 14, 405-415. https://doi.org/10.1207/ s15327663jcp1404_9 
Morrison, T. G., Kenny, P., \& Harrington, A. (2005). Modern prejudice toward gay men and lesbian women: Assessing the viability of a measure of modern homonegative attitudes within an Irish context. Genetic, Social, and General Psychology Monographs, 131, 219- 250. https:// doi.org/10.3200/MONO.131.3.219-250

Morrison, M. A., \& Morrison, T. G. (2002). Development and validation of a scale measuring modern prejudice toward gay men and lesbian women. Journal of Homosexuality, 43, 15-37. https://doi.org/10.1300/j082v43n02_02

Murphy, C., MacCarthaigh, S., \& BarnesHolmes, D. (2014). The Implicit Relational Assessment Procedure and attractiveness bias: Directionality of bias and influence of gender of participants. International Journal of Psychology and Psychological Therapy, 14, 333-351.

Nicholson, E., \& Barnes-Holmes, D. (2012). The Implicit Relational Assessment Procedure (IRAP) as a measure of spider fear. The Psychological Record, 62, 263-278. https://doi. org/10.1007/BF03395801

Nolan, J., Murphy, C., \& Barnes-Holmes, D. (2013). Implicit Relational Assessment Procedure and body-weight bias: Influence of gender participants and targets. The Psychological Record, 63, 467-488. https://doi.org/10.11133/j. tpr.2013.63.3.005

Power, P., Barnes-Holmes D., Barnes-Holmes, Y., \& Stewart, I. (2009). The Implicit Relational Assessment Procedure (IRAP) as a measure of implicit relative preferences: A first study. The Psychological Record, 59, 621-640. https://doi. org/10.1007/BF03395684

Rabelo, L. Z., Bortoloti, R., \& Souza, D. H. (2014). Dolls are for girls and not for boys: Evaluating the appropriateness of the implicit relational assessment procedure for school-age children. The Psychological Record, 64, 71-77. https://doi. org/10.1007/s40732-014-0006-2

Rönspies, J., Schmidt, A. F., Melnikova, A., Krumova, R., Zolfagari, A., \& Banse, R. (2015). Indirect measurement of sexual orientation: Comparison of the implicit relational assessment procedure, viewing time, and choice reaction time tasks. Archives of Sexual Behavior,44, 1-32. https://doi. org/10.1007/s10508-014-0473-1
Scheel, M. H., Fischer, L. A., McMahon, A. J., Mena, M. M., \& Wolf, J. E. (2011). The Implicit Relational Assessment Procedure (IRAP) as a measure of women's stereotypes about gay men. Current Research in Social Psychology, 18, Article 2.

Silva, M. V. M. (2017). Violência LGBTFóbicas no Brasil: Dados da Violência. Ministério dos Direitos Humanos. Recuperado de:

http://www.mdh.gov.br/biblioteca/consultorias/ lgbt/violencia-lgbtfobicas-no-brasil-dados-da-violencia

Snowden, R. J., Wichter, J., \& Gray, N. S. (2008). Implicit and explicit measurements of sexual preference in gay and heterosexual men: A comparison of priming techniques and the implicit association task. Archives of Sexual Behavior, 37, 558-565. https://doi.org/10.1007/ s10508-006-9138-Z

Steffens, M. C. (2005). Implicit and explicit attitudes toward lesbians and gay men. Journal of Homosexuality, 49, 39-66. https://doi. org/10.1300/J082v49n02_03

Steffens, M. C., \& Buchner, A. (2003). Implicit Association Test: Separating transsituationally stable and variable components of attitudes toward gay men. Experimental Psychology, 50, 3348. https://doi.org/10.1027//1618-3169.50.1.33

Szymanksi, J., \& O’Donohue, W. (1995). Fear of spiders questionnaire. Journal of Therapy and Experimental Psychiatry, 26, 31-34. https://doi. org/10.1016/0005-7916(94)00072-t

Timmins, L., Barnes-Holmes, D., \& Cullen, C. (2016). Measuring implicit sexual response biases to nude male and female pictures in androphilic and gynephilic men. Archives of Sexual Behavior, 45, 829-841. https://doi.org/10.1007/ s10508-016-0725-3

\section{Informações do Artigo}

Histórico do artigo:

Submetido em: 11/01/2021

Primeira decisão editorial: 07/04/2021

Aceito em: 30/04/2021

Editor: William F. Perez 